\title{
Isolamento, identificazione e sensibilità ai farmaci antimicrobici di due ceppi di Mycobacterium marinum da fish tank granuloma
}

\author{
Barbara Saddi', Francesco Usai', Alessandra Burgio', Caterina Ferreli², \\ Alessandro De Logu', Aldo Vito Carluccio' \\ 'Laboratorio di Analisi Chimico-Cliniche e Microbiologia, Ospedale SS. Trinità, Cagliari \\ ${ }^{2}$ Dipartimento di Scienze Mediche Internistiche - Clinica Dermatologica, Università di Cagliari \\ ${ }^{3}$ Dipartimento di Scienze Chirurgiche e Trapianti d’Organo, Sezione di Microbiologia e Virologia, Università di Cagliari
}

Isolation, identification and antimicrobial susceptibilty of two strains of Mycobacterium marinum from fish tank granuloma

Key Word: Mycobacterium marinum, fish tank granuloma, drug-sucepibility testing

\section{SUMMARY}

Mycobacterium marinum is an emerging pathogen responsible for cutaneous infections related to the handling of domestic aquariums. Two distinct strains of M. marinum have been isolated from as many cases of fish tank granuloma. Despite the ability of both strains to grow at $37^{\circ} \mathrm{C}$, the detection of their growth by the automated BACTEC $9000 \mathrm{MB}$ system, was delayed or not happened at all in comparison with the growth of mycobacterium culture in the Löwenstein-Jensen medium. The different antibiotic susceptibility pattern showed by $M$. marinum in several studies, required to perform suscepibility testing of two clinical isolates. Both strains were resistant to isoniazid, imipenem, cefoxitine, susceptible to rifampicin, ethambutol, clarithromycin, ciprofloxacin and moderately susceptible to doxycycline.

\section{INTRODUZIONE}

Mycobacterium marinum è un micobatterio atipico, fotocromogeno, classificato nel gruppo I di Runyon. In coltura, cresce in 7-14 giorni alla temperatura ottimale di $32^{\circ} \mathrm{C}$, formando colonie prevalentemente di tipo $\mathrm{S}$. È causa di infezioni tubercolari sistemiche nei pesci marini e di acqua dolce (2). Nell'uomo, invece, le infezioni sostenute da M. marinum sono principalmente cutanee, conseguenti alla presenza di piccole abrasioni, ed associate al contatto con acque contaminate, dolci o salate, o con animali acquatici. Tali infezioni sono state descritte per la prima volta in individui frequentatori di piscine (swimming pool granuloma), ed attualmente sono segnalate con frequenza sempre maggiore in soggetti esposti al contatto con acquari (fish tank granuloma) o con allevamenti di pesci $(16,17,20,21$,$) . Tipicamente, le lesioni$ cutanee compaiono all'estremità degli arti dopo 2-3 settimane di incubazione, e sono caratterizzate dalla presenza di formazioni papulo-nodoulari, con evoluzione verso forme verrucose ed ulcerate, od anche ascessi. In alcuni casi, gli elementi nodulari proliferano lungo la via di drenaggio dei vasi linfatici, con una distribuzione di tipo sporotricoide. Le forme extracutanee, quali tenosinoviti, osteomieliti, artriti, sono meno comuni; rare forme disseminate sono state descritte per lo più in pazienti immunocompromessi $(6,7,19)$.
La terapia non è ben definita: rifampicina, etambutolo, claritromicina, tetracilcline, e cotrimoxazolo, da soli o in combinazione, sono i farmaci più usati nel trattamento delle infezioni da $M$. marinum, ma in alcuni casi sono stati impiegati con successo anche i fluorochinoloni $(11,12,17,18)$. La durata del trattamento è variabile da 2 settimane a 18 mesi, ma è importante che venga continuata per 2-3 mesi dopo la risoluzione delle manifestazioni cliniche (23).

Negli USA, la frequenza di isolamento di $M$. marinum è bassa, dal momento che meno dell' $1 \%$ degli isolati clinici di micobatteri appartiene a questa specie, ma l'incidenza dell'infezione è variabile in relazione all'area geografica $(12,15)$. In Francia, M. marinum è considerato un patogeno emergente di infezioni associate all'hobby degli acquari, e tra il 1996 e il 1998 ne sono stati documentati 63 casi (3). In Italia, le segnalazioni di infezioni da $M$. marinum sono relativamente rare, anche per la mancanza di un sistema di sorveglianza a livello nazionale $(1,5,8,9,10,13,22,24)$.

Riportiamo due casi di infezione cutanea da $M$. marinum, giunti all'attenzione del Laboratorio di Microbiologia dell'Ospedale SS. Trinità di Cagliari, nel novembre del 2002. Entrambi i casi sono stati riscontrati in soggetti immunocompetenti proprietari di acquari domestici tropicali, in 
cui erano soliti immergere le mani e gli avambracci nudi, durante le periodiche procedure di pulizia.

\section{DESCRIZIONE DEI CASI CLINICI}

Caso 1. Paziente di 24 anni, di sesso maschile, proprietario di un acquario tropicale di acqua dolce. All'anamnesi il paziente riferiva la morte di alcuni pesci avvenuta alcune settimane prima dell'esordio delle manifestazioni cliniche, e preceduta dalla comparsa di lesioni deformanti.

Al momento della prima osservazione, nel novembre del 2002, il paziente presentava su un dito della mano sinistra, numerosi elementi nodulari di colorito rosso-violaceo, delle dimensioni di una piccola lenticchia, alcuni isolati, altri confluenti in placche di maggiori dimensioni, ricoperti da cute liscia e lucida, che in alcuni punti mostrava la presenza di crosticine giallastre. Analoghi elementi nodulari erano presenti anche in corrispondenza del polso e dell'avambraccio, ricoperti da cute lievemente eritematosa ed alopecica. Dopo la diagnosi microbiologica, il paziente è stato trattato con doxiciclina alla dose di $100 \mathrm{mg}$ p.o. 2 volte al giorno per 15 giorni, con remissione delle lesioni cutanee. Tuttavia, a distanza di tre mesi, si osservava una recidiva delle manifestazioni cliniche, cui ha fatto seguito un nuovo ciclo di terapia della durata di 30 giorni.

Caso 2. Paziente di 39 anni, di sesso maschile, proprietario di un acquario tropicale di acqua dolce. Anche in questo caso, veniva riferita la morte di alcuni pesci riscontrata alcuni mesi prima della comparsa delle manifestazioni cliniche.

Al momento della prima osservazione, nel novembre del 2002, il paziente presentava sulla regione metacarpale della mano destra e sulle dita, alcune formazioni ascessuali del diametro di $1-3 \mathrm{~cm}$, circondate da un alone di infiltrazione rosso-violaceo, dalle quali fuoriusciva spontaneamente del materiale purulento. Su una delle dita erano anche presenti alcune piccole lesioni nodulari, con al centro una piccola crosta.

L'articolazione interfalangea del I dito si presentava notevolmente aumentata di volume, fortemente dolente, con limitazione funzionale. Due formazioni nodulari, non adese alla cute, e delle dimensioni di un piccolo fagiolo, erano riscontrabili sul versante radiale del braccio e dell'avambraccio. Le stazioni linfonodali epitrocleali ed ascellari erano indenni e non erano presenti altri segni di interessamento generale. Dopo la diagnosi microbiologica, fu instaurata una terapia con rifabutina alla dose di $150 \mathrm{mg}$ p.o. 2 volte al giorno, etambutolo alla dose di $20 \mathrm{mg} / \mathrm{Kg} /$ die p.o. in 3 somministrazioni giornaliere, claritromicina alla dose di $500 \mathrm{mg}$ p.o. 2 volte al giorno. Il trattamen- to è stato protratto per 5 mesi, con completa risoluzione delle lesioni cutanee.

\section{MATERIALI E METODI}

\section{Esame microscopico, isolamento colturale,} identificazione

Il ceppo di M. marinum del caso $1(\mathrm{Mm} 1)$ è stato isolato da una biopsia cutanea, prelevata dalle lesioni del dito della mano sinistra e trasportata in laboratorio all'interno di un urobox. L'osservazione microscopica, previa colorazione di Ziehl-Neelsen, mostrava la presenza di rare forme bastocellari, lunghe e grosse, acido-alcool resistenti. La biopsia, finemente sminuzzata e dispersa in alcuni $\mathrm{ml}$ di soluzione fisiologica, è risultata contaminata da altre specie batteriche alla semina su agar sangue; dopo decontaminazione con $\mathrm{NaOH}$ al $2 \%$, il materiale è stato seminato $(0.5 \mathrm{ml}$ circa) in un flacone BACTEC MYCO/FSputa (Sistema BACTEC 9000MB; Becton Dickinson), e quindi in due tubi di LöwensteinJensen (LJ), incubati rispettivamente a $37^{\circ} \mathrm{C}$ e a temperatura ambiente, non disponendo al momento di un termostato a temperatura inferiore ai $37^{\circ} \mathrm{C}$. Dopo circa due settimane di incubazione, nel tubo di LJ incubato a temperatura ambiente ed esposto alla luce, è stato possibile evidenziare la crescita di rare colonie pigmentate di giallo, che all'esame microscopico, dopo colorazione di Ziehl-Neelsen, mostravano la presenza di bastoncelli acido-alcool resistenti.

Nelle subcolture tenute al buio, la produzione della pigmentazione gialla si osservava solo dopo alcune ore di esposizione alla luce, consentendo di classificare il ceppo in esame tra i Micobatteri fotocromogeni (Guppo I di Runyon), e di identificarlo, tramite prove biochimiche, come Mycobacterium marinum (tabella 1). Nessuna crescita è stata rilevata nel tubo di LJ e nel flacone BACTEC MYCO/F-Sputa, incubati per 8 settimane a $37^{\circ} \mathrm{C}$, sebbene, nelle subcolture, il ceppo fosse in grado di crescere anche a $37^{\circ} \mathrm{C}$.

Il ceppo di M. marinum del caso $2(\mathrm{Mm} 2)$, è stato isolato da un materiale purulento, prelevato con una siringa dalle lesioni della mano destra e trasportato come tale al laboratorio di Microbiologia. L'esame microscopico, dopo colorazione di Ziehl-Neelsen, evidenziava la presenza di numerose forme bastoncellari, acido-alcool resistenti. Il campione, risultato privo di contaminazione microbica alla semina su agar sangue, è stato inoculato direttamente, previa diluizione con alcuni $\mathrm{ml}$ di acqua distillata, in un flacone BACTEC MYCO/F-Sputa, e quindi in due tubi di Löwenstein-Jensen (LJ), incubati rispettivamente a $37^{\circ} \mathrm{C}$ e a temperatura ambiente. Dopo circa 10 giorni di incubazione, nei tubi di LJ si osservava 
Tabella I

\begin{tabular}{|c|c|c|c|c|c|c|c|c|c|c|c|c|c|c|c|}
\hline & 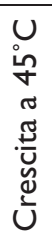 & 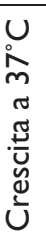 & 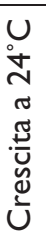 & 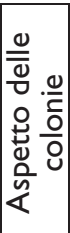 & 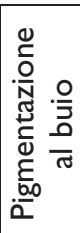 & 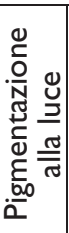 & 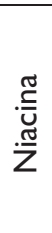 & 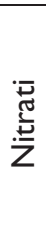 & 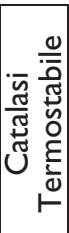 & 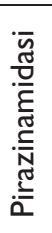 & $\frac{\bar{y}}{\frac{\bar{d}}{\tilde{w}}}$ & 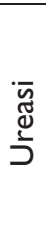 & 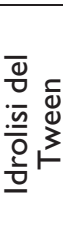 & 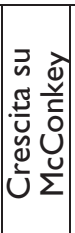 & 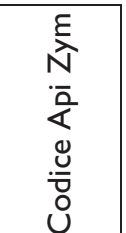 \\
\hline $\mathrm{Mml}$ & 一 & + & + & S & - & + & - & - & $+*$ & - & - & + & + & - & 6706002 \\
\hline $\mathrm{Mm} 2$ & - & + & + & $S$ & - & + & - & - & - & + & - & + & + & - & 6706002 \\
\hline
\end{tabular}

*Debole rispetto al controllo positivo M. kansasii

una patina di crescita, pigmentata di giallo nel tubo tenuto alla luce a temperatura ambiente, ma priva di pigmentazione nel tubo incubato al buio a $37^{\circ} \mathrm{C}$. L'esame microscopico confermava in entrambi i casi la presenza di bastoncelli acidoacool resistenti. Tramite prove biochimiche e colturali, il ceppo in esame è stato, quindi, identificato come Mycobacterium marinum (tabella 1). Nel BACTEC, il rilevamento della crescita del micobatterio avveniva circa un mese dopo la semina. L'esame microscopico di una sospensione ottenuta dal filtro dell'acquario del paziente, mostrava numerosissime formazioni bastoncellari acido-alcool resistenti. Tuttavia, il tentativo di isolare dal materiale il $M$. marinum, ha dato esito negativo a causa dello sviluppo in coltura di altri Micobatteri a rapida crescita, uno dei quali è stato identificato come M. fortuitum.

\section{SENSIBILITÀ AI FARMACI ANTIMICROBICI}

La sensibilità a isoniazide, rifampicina, etambutolo, ciprofloxacina e doxiciclina, è stata determinata con il metodo proporzionale su terreno Middlebrook 7H11, supplementato con il 10\% di $\mathrm{OADC}$, e distribuito in piastre suddivise in quadranti. Gli antibiotici in esame sono stati incorporati nel terreno in modo da ottenere concentrazioni finali, corrispondenti ai breakpoints di sensibilità. Da una subcoltura di 7 giorni, cresciuta su LJ, è stata preparata una sospensione del ceppo in esame, con una torbidità equivalente allo standard McFarland 1 (circa $10^{8} \mathrm{CFU} / \mathrm{ml}$ ). Diluizioni 1/100 e 1/10.000 di tale sospensione, sono state inoculate $(50 \mu 1)$ sui quadranti contenenti il terreno con $\mathrm{i}$ singoli farmaci e sui corrispondenti quadranti di controllo, con terreno senza farmaco. Le piastre sono state incubate per 6 giorni a $30^{\circ} \mathrm{C}$, in atmosfera arricchita in $\mathrm{CO}_{2}$ al 5\%. La sensibilità al farmaco è definita dall'assenza di crescita o da una crescita con un numero di colonie $<1 \%$ di quelle cresciute sul terreno di controllo; sono state prese in considerazione anche le microcolonie appena visibili ad occhio nudo.
Entrambi i ceppi sono risultati resistenti all'isoniazide ( $\mathrm{MIC} \geqslant 0,2 \mu \mathrm{g} / \mathrm{ml})$, sensibili alla rifampicina $(\mathrm{MIC} \leqslant 1 \mu \mathrm{g} / \mathrm{ml}), \quad$ all' etambutolo $(\mathrm{MIC} \leqslant 3,5 \mu \mathrm{g} / \mathrm{ml} ; \mathrm{S} \leqslant 7,5 \mu \mathrm{g} / \mathrm{ml})$ e alla ciprofloxacina $(\mathrm{MIC} \mu 1 \mathrm{mg} / \mathrm{ml})$, e con sensibilità intermedia alla doxiciclina ( $\mathrm{MIC} \leqslant 8 \mu \mathrm{g} / \mathrm{ml}$ ), secondo i breakpoints di questo farmaco raccomandati dall'NCCLS per i batteri aerobi $(S \leqslant 4-R \geqslant 16)$.

La sensibilità alla claritromicina, all'imipenem e alla cefoxitina, è stata determinata con il metodo dell'E-test (AB-Biodisk; Solna, Sweden). Le strisce E-test di ogni singolo farmaco sono state applicate sulla superficie di piastre di 7H11, inoculate, usando un tampone sterile, con una sospensione del ceppo in esame, di torbidità equivalente allo standard McFarland 1. Le piastre sono state incubate per 6 giorni a $30^{\circ} \mathrm{C}$, in $\mathrm{CO}_{2}$ al $5 \%$. Il valore della MIC è letto nel punto in cui la zona di inibizione della crescita interseca la scala di lettura graduata presente sulla faccia della striscia.

La MIC della claritromicina è risultata uguale a $0,5 \mu \mathrm{g} / \mathrm{ml}$ per entrambi i ceppi, che, quindi, devono essere considerati sensibili al farmaco, secondo i breakpoints raccomandati dall'NCCLS per i batteri aerobi $(S \leqslant 2-R \geqslant 8)$. All'imipenem e alla cefoxitina, la resistenza era totale (nessun alone di inibizione). Analoghi risultati sono stati ottenuti usando per 1'E-test, l'agar Mueller-Hinton con sangue di montone al 5\%, al posto del $7 \mathrm{H} 11$.

\section{DISCUSSIONE}

Il $M$. marinum è causa di infezioni cutanee riscontrate con frequenza sempre maggiore in soggetti esposti al contatto con acquari domestici. La conoscenza clinica dell'infezione e dei fattori di rischio correlati sono importanti per una diagnosi precoce. Tuttavia, l'isolamento colturale del microrganismo è indispensabile per una conferma eziologica della diagnosi, e per instaurare un'adeguata terapia sulla base della sensibilità in vitro ai farmaci antimicrobici. Il ritardo o l'assenza della crescita da noi osservati nel BACTEC 9000MB, potrebbero essere attribuiti all'incubazione ad una temperatura $\left(37^{\circ} \mathrm{C}\right)$ diversa da quella ottimale 
$\left(32^{\circ} \mathrm{C}\right)$ richiesta per la crescita del M. marinum. Questa circostanza evidenzia la necessità di incubare le colture ad una temperatura inferiore ai $37^{\circ} \mathrm{C}$, in caso di sospetta infezione cutanea o sottocutanea da micobatteri atipici. Attualmente non esistono dei metodi standardizzati per il test di sensibilità antibiotica del $M$. marinum. L'E-test rappresenta una metodica di facile esecuzione, utilizzata in diversi studi per la determinazione delle MIC del M. marinum (14). Tuttavia, la sua correlazione con il metodo delle diluizioni in agar di riferimento, è risultata buona solo per certi farmaci come la minociclina e la rifampicina, ma bassa per altri come la claritromicina e l'etambutolo; inoltre con alcuni antimicrobici, come il cotrimoxazolo, sono state riscontrate delle difficoltà di lettura, a causa della presenza di aloni di inibizione non ben definiti $(4,25)$. In particolare, le MIC della claritromicina determinate con il metodo delle diluizioni in agar sono risultate, in genere, maggiori di 1-3 diluizioni rispetto a quelle misurate con l'E-test; ma nonostante queste differenze l'interpretazione della sensibilità al farmaco non veniva compromessa. In conclusione, il diverso pattern di sensibilità antibiotica esibito dal M. marinum in differenti studi, sottolinea la necessità di eseguire un antibiogramma su i singoli isolati clinici, ma l'utilizzo dell'E-test deve essere valutato attentamente per ogni singolo farmaco.

\section{BIBLIOGRAFIA}

1. Amerio P, Carmenini S, De Mori F, Santone R. Micobatteriosi atipica da Mycobacterium marinum. $G$ Ital Dermatol Venereol 1990; 125: 147-9.

2. Aronson JD. Spontaneous tuberculosis in salt fish. $J$ Infect Dis 1926; 39: 315-20.

3. Aubry A, Chosidow O, Caumes E, Robert J, Cambau E. Sixty-three cases of Mycobacterium marinum infection: clinical features, treatment, and antibiotic susceptibility of causative isolates. Arch Intern Med 2002; 162: 1746-52

4. Aubry A, Jarlier V, Escolano S, Truffot-Pernot C, Cambau E. Antibiotic susceptibility pattern of Mycobacterium marinum. Antimicrob Agents Chemother 2000; 44: 3133-6.

5. Barba A, Tessari G, Schena D. Skin infection due to Mycobacterium marinum in a renal transplant recipient. J Nephrol 1996; 9: 78-80.

6. Barton A, Bernstein RM, Struthers JK. Mycobacterium marinum infection causing septic arthritis and osteomyelitis. Br J Rheumatol 1997; 36: 1207-9.

7. Bodemer C, Durand C, Blanche S, Teillac D, de Prost Y. [Disseminated Mycobacterium marinum infection]. Ann Dermatol Venereol 1989; 116: 842-3.

8. Califano L, Cannavò SP, Malara G. Verrucous Nodule of the Finger. Arch Dermatol 1998; 134: 365-70.

9. Castiglia N, Lorenzoni A, Molicotti P, Satta S, Cherchi GB. Microrganismi di raro riscontro: descrizione di un caso di micobatteriosi cutanea da
Mycobacterium marinum nel Nord-Sardegna. Dal Congresso Regionale Sardo dell'AMCLI; Cagliari, 5 maggio 2000.

10. Cavalcanti P, Filia MA, Cannataro AM, Dodaro S, Greco LM, Magnano C. Riscontro di M. marinum in un soggetto immunocompetente. Microbiologia Medica 2002; 17: 170

11. Donta ST, Smith PW, Levitz RE, Quintiliani R. Therapy of Mycobacterium marinum infections. Use of tetracyclines vs rifampin. Arch Intern Med 1986; 146: 902-4.

12. Edelstein H. Mycobacterium marinum skin infections. Report of 31 cases and review of the literature. Arch Intern Med 1994;154:1359-64.

13. Fioroni AM, Brandani M, Japadre N, Bruno G. Rapido isolamento di Mycobacterium marinum in un caso di granuloma dell'acquario (fish tank granuloma). Dal Congresso dell'AMCLI; Rimini 12-15 settembre 2000.

14. Flynn CM, Kelley CM, Barrett MS, Jones RN. Application of the E-test to the antimicrobial susceptibility testing of Mycobacterium marinum clinical isolates. J Clin Microbiol 1997; 35: 2083-6.

15. Good R, Snider D. Isolation of nontuberculous mycobacteria in the United States. J Infect Dis 1979; 146: 829-33.

16. Gray SF, Stanwell SR, Reynolds NJ, Williams EW. Fish tank granuloma. BMJ 1990; 300: 1069-70.

17. Huminer D, Pitlik SD, Block C, Kaufman L, Amit S, Rosenfeld JB. Aquarium-borne Mycobacterium marinum skin infection. Report of a case and review of the literature. Arch Dermatol 1986; 122: 698-03.

18. Iijima S, Saito J, Otsuka F. Mycobacterium marinum skin infection successfully treated with levofloxacin. Arch Dermatol 1997; 133: 947-9.

19. Kishihara Y, Nakashima K, Nukina H, Hayashi J, Kashiwagi S. Two cases of acquired immunodeficiency syndrome with disseminated non-tuberculous mycobacterial infection. Kansenshogaku Zasshi 1993; 67: 1223-7.

20. Norden A, Linell F. A new type of pathogenic mycobacterium. Nature 1951; 168: 826

21. Philpott JA, Woodburne AR, Philpott OS, Schaefer WB, Mollohan CS. Swimming pool granuloma. Arch. Dermatol 1963; 88: 158-62.

22. Puiatti P, Alberico G, Cotilli G, Salvai M, Goitre M. Sporotrichoid infection. Two cases. G Ital Dermatol Venereol 1990; 125: 349-52.

23. Tobin EH, Jih WW. Sporotrichoid lymphocutaneous infections: etiology, diagnosis and therapy. Am Fam Physician 2001; 63: 326-32.

24. Vincenzi C, Bardazzi F, Tosti A, Varotti C, Morganti L. Fish tank granuloma: report of a case. Cutis 1992; 49: 275-6.

25. Werngren J, Olsson-Liljequist B, Gezelius L, Hoffner SE. Antimicrobial susceptibility of Mycobacterium marinum determined by E-test and agar dilution. Scand J Infect Dis 2001; 33: 585-8.

\section{Aldo Vito Carluccio}

Laboratorio di Analisi Chimico-Cliniche e Microbiologia

Ospedale SS. Trinità

Via Is Mirrionis 92 - 09122 Cagliari

Tel.: 070 / 6095934; Fax: 070 / 6095894

E-mail: aldo.carluccio@tiscali.it 